Supporting information

\title{
Synthesis and high hydrogen storage capacity of zeolite-like carbons nanocast using as-synthesised zeolite templates
}

\author{
A. Pacula ${ }^{a, b}$, R. Mokaya*a \\ ${ }^{a}$ School of Chemistry, University of Nottingham, University Park, Nottingham NG7 2RD, U.K. \\ Tel.+44 115 8466174; Fax: +440115 9513562.E-mail: r.mokaya@,nottingham.ac.uk \\ ${ }^{b}$ Institute of Catalysis and Surface Chemistry, Polish Academy of Sciences, ul. Niezapominajek \\ 8, 30-239 Kraków, Poland.
}




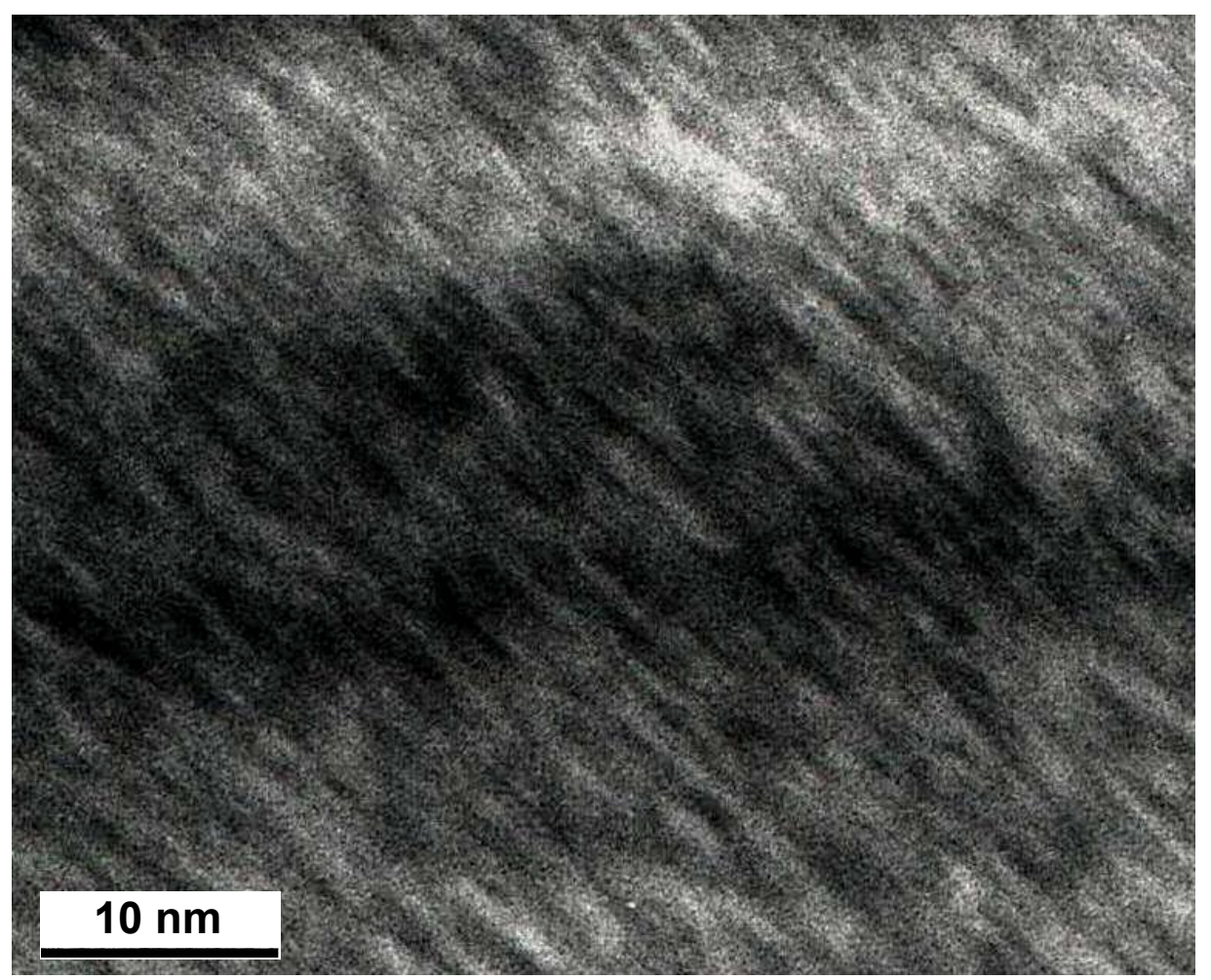

Supporting Figure 1S. Representative TEM image of zeolite-templated carbon prepared at CVD temperature of $850{ }^{\circ} \mathrm{C}$. 
Table S1. Textural properties, elemental composition and hydrogen uptake of carbons prepared via CVD at various temperatures using calcined zeolite $\beta$ as template.

\begin{tabular}{lllll}
\hline $\begin{array}{l}\text { CVD Temperature } \\
\left({ }^{\circ} \mathrm{C}\right)\end{array}$ & $\begin{array}{l}\text { N content } \\
(\mathrm{wt} \%)\end{array}$ & $\begin{array}{l}\text { Surface area } \\
\left(\mathrm{m}^{2} / \mathrm{g}\right)^{\mathrm{a}}\end{array}$ & $\begin{array}{l}\text { Pore Volume } \\
\left(\mathrm{cm}^{3} / \mathrm{g}\right)^{\mathrm{b}}\end{array}$ & $\begin{array}{l}\text { Hydrogen Uptake } \\
(\mathrm{wt} \%)^{\mathrm{c}, \mathrm{d}}\end{array}$ \\
\hline $800^{*}$ & 3.0 & $1928(732)$ & $1.79(0.32)$ & $2.4(0.8)$ \\
$850^{*}$ & 3.2 & $2272(1014)$ & $1.96(0.45)$ & $3.1(1.2)$ \\
$900^{*}$ & 4.8 & $2176(991)$ & $1.67(0.43)$ & 3.6 \\
& & & & \\
$\mathrm{CA} 800^{* *}$ & & $2191(1213)$ & $1.79(0.56)$ & 3.9 \\
$\mathrm{CA} 850^{* *}$ & & $3189(1529)$ & $2.41(0.71)$ & 6.0 \\
$\mathrm{CB} 850^{* *}$ & & $2611(1150)$ & $1.74(0.79)$ & 5.5 \\
$\mathrm{CB} 850 \mathrm{~h}^{* * \mathrm{e}}$ & & $3150(2397)$ & $1.95(1.13)$ & 6.9
\end{tabular}

${ }^{a}$ Values in parenthesis are micropore surface area. ${ }^{b}$ Values in parenthesis are micropore volume. ${ }^{\mathrm{c}}$ Hydrogen uptake capacity at $-196{ }^{\circ} \mathrm{C}$ and 20 bar. ${ }^{\mathrm{d}}$ Values in parenthesis are hydrogen uptake capacity at $-196{ }^{\circ} \mathrm{C}$ and 1 bar. ${ }^{~}$ Obtained from sample CB850 after further heat treatment (see ref. 7 for details).

* Yang, Z.; Xia, Y.; Mokaya, R. Microporous Mesoporous Mater. 2005, 86, 69 (ref. 9)

(*These samples are identical to those reported in Table 1, except for the use of calcined rather than as-synthesised zeolite $\beta$ as template. Comparison with data in Table 1 highlights the singular benefit of using as-synthesised zeolite as template.)

** Yang, Z.; Xia, Y.; Mokaya, R. J. Am. Chem. Soc. 2007, 129, 1673 (ref. 7)

(**These samples are prepared via modified CVD routes that enhance microporosity.) 